\title{
Building a Better Mousetrap: Replacing Subjective Writing Rubrics with More Empirically-Sound Alternatives for EFL Learners
}

\author{
Andrew D. Schenck, Eoin Daly* \\ English Education, Department of Liberal Arts Education (LAEC), Ju Si-Gyeong College, Pai Chai University, \\ Daejeon, South Korea \\ Email: Schenck@hotmail.com, ${ }^{*}$ eointeacher@yahoo.com
}

Received October $2^{\text {nd }}, 2012$; revised November $5^{\text {th }}, 2012$; accepted November $9^{\text {th }}, 2012$

\begin{abstract}
Although writing rubrics can provide valuable feedback, the criteria they use are often subjective, which compels raters to employ their own tacit biases. The purpose of this study is to see if discreet empirical characteristics of texts can be used in lieu of the rubric to objectively assess the writing quality of EFL learners. The academic paragraphs of 38 participants were evaluated according to several empirically calculable criteria related to cohesion, content, and grammar. Values were then compared to scores obtained from holistic scoring by multiple raters using a multiple regression formula. The resulting correlation between variables $(R=.873)$ was highly significant, suggesting that more empirical, impartial means of writing evaluation can now be used in conjunction with technology to provide student feedback and teacher training.
\end{abstract}

Keywords: Writing Rubrics; Writing Evaluation; Cohesion; Grammar; Word Frequency

\section{Introduction}

Several studies recognize the efficacy of the rubric as a means to score writing and provide feedback (Cope, Kalantzis, McCarthey, Vojak, \& Kline, 2011; Mansilla, Duraisingh, Wolfe, \& Haynes, 2009; Peden \& Carroll, 2008). A study by Beyreli and Ari (2009), for example, found that it could be accurately used to assess ten properties related to structure, language, and organization with a fair degree of inter-rater reliability (from $65 \%$ to $81 \%$ ). Another study revealed that it could be used to evaluate writing holistically, regardless of the participants' L1 (Sévigny, Savard, \& Beaudoin, 2009). Recent adaptations of the rubric have even discovered the potential to increase formative feedback through the use of both technology and self-assessment strategies (Cope, Kalantzis, McCarthey, Vojak, \& Kline, 2011; Peden \& Carroll, 2008).

While rubrics can provide a systematic means to evaluate student writing, their reliability and validity can be questionable. This is exemplified by recent studies, which reveal that rater bias and invalidity of writing assessments are negatively impacting summative student evaluation (Graham, Hebert, \& Harris, 2011: p. 10; Johnson \& VanBrackle, 2012). To overcome these shortcomings, educators have advocated the use of more authentic assessment methods such as self-assessment checklists, writing conferences, and writing portfolios (Schulz, 2009).

Current problems with reliability and validity of the writing rubric may be caused by the subjectivity of rubric criteria. As pointed out by Fang and Wang (2011), such criteria contain expressions such as "exceptionally clear", "effectively organized", "carefully chosen", and "strong control", which force teachers to "rely on their own intuition and discursive knowledge in mak-

"Corresponding author. ing judgment calls" (Fang \& Wang, 2011: p. 148). In reality, this use of vague, subjective descriptors for different categories of writing reflect a deficiency in understanding of what constitutes good writing. Exploration of more objective, empirical measures of writing quality may improve this understanding, thereby allowing for the development of more effective evaluation techniques (Sévigny, Savard, \& Beaudoin, 2009). The purpose of this study, therefore, is to examine multiple empirical criteria and their influence on overall writing quality.

\section{Disparities between Writing Rubrics}

Many educators have attempted to increase the validity and reliability of writing evaluation through the development of rubrics. Although they are a useful step forward, key limitations remain. One of the largest problems with such rubrics is the subjectivity and ambiguity of language they contain. Holistic rubrics, for example, which rely upon general impressions of quality based upon descriptors contained within each proficiency level, often contain vague language which masks the significance of results and lessens the potential for washback (Brown, 2004). Consider the following examples contained within levels 4 and 5 of the Test of English as a Foreign Language (TOEFL) rubric for academic writing (Educational Testing Service, 2008):

\section{Criteria for Rubric Level 4}

1) Addresses the topic and task well, though some points may not be fully elaborated.

2) Is generally well organized and well developed, using appropriate and sufficient explanations, exemplifications, and/or details.

3) Displays unity, progression, and coherence, though it may contain occasional redundancy, digression, or unclear connections. 
4) Displays facility in the use of language, demonstrating syntactic variety and range of vocabulary, though it will probably have occasional noticeable minor errors in structure, word form, or use of idiomatic language that do not interfere with meaning.

\section{Criteria for Rubric Level 5}

1) Effectively addresses the topic and task.

2) Is well organized and well developed, using clearly appropriate explanations, exemplifications, and/or details.

3) Displays unity, progression, and coherence.

4) Displays consistent facility in the use of language, demonstrating syntactic variety, appropriate word choice, and idiomaticity, though it may have minor lexical or grammatical errors.

As revealed by the words highlighted in bold text, criteria within levels four and five can be decidedly subjective. Expressions such as "effectively addresses the topic" or "addresses the topic and task well", for example, cannot be assigned an empirical value, since they rely primarily on the opinion of an evaluator. Raters must use their own intuition to interpret whether the text is "effective" by using their unique personal experiences and cultural backgrounds. Other terms, such as "appropriate", "sufficient", "occasional", and "probably", are also ambiguous, and may be interpreted differently depending upon personal characteristics of the reviewer. It is this ambiguity that requires extensive training to attain an acceptable level of interrater reliability (Brown, 2004). Due to such problems with holistic writing rubrics, it is imperative that more objective means of evaluating writing are developed to increase reliability and decrease the need for extensive training of multiple raters.

In addition to problems with subjectivity, rubric criteria often evaluate disparate traits, making assertions of validity problematic. The TOEFL IBT rubric, for example, evaluates factors such as organization, unity, coherence, grammar, and idiomatic language of the academic essay genre (Educational Testing Service, 2008), while the American Council on the Teaching of Foreign Languages (ACTFL) evaluates multiple genres in conjunction with factors such as fluency, use of low-frequency structures, and vocabulary (American Council on the Teaching of Foreign Languages, 2012). Yet another writing rubric designed by Peregoy \& Boyle (2005) includes sentence variety in addition to elements included in both the TOEFL and ACTFL rubrics.

In summary, criteria within rubrics have a great deal of ambiguity and disparity, which make determinations of validity and reliability more problematic. Raters are compelled to interpret criteria differently, leading to the introduction of personal biases during the writing evaluation process. This behavior is exemplified through a study by Hunter and Docherty (2011), which reveals that raters use their own tacit expectations to interpret and evaluate aspects of writing structure, content, and expression. Because of problems with rubrics and associated rater behaviors, more objective empirical measures of writing quality are needed. Such measures can increase validity and reliability by ensuring that multiple raters assess precisely what is prescribed within set criteria. Moreover, the use of these measures can allow for extensive automation of writing evaluation. While some empirical measures of writing quality have now been developed, they continue to yield EFL writing scores that differ significantly from those assigned by human raters (Chodorow \& Burstein, 2004; Weigle, 2010). More study is needed to understand how empirical criteria may be used to evaluate the overall writing quality of diverse learners. The purpose of this study, therefore, is to see if multiple empirical measures can be used to accurately assess the quality of academic writing composed by EFL learners.

\section{Research Questions}

1) Can empirical methods of text evaluation (e.g., cohesion, content, and grammatical accuracy) be collectively used in lieu of a holistic scale to accurately rate the writing of EFL learners?

2) How can empirical measures of writing quality be used to improve evaluation and education in an EFL setting?

\section{Method}

The purpose of this quasi-experimental study was to see if writing quality could be accurately assessed through using empirical measures of EFL writing. Due to the complexity of developing measures for multiple types of discourse, only one genre, that of academic writing, was examined within this study. Traditional evaluation methods, which required multiple raters and a holistic scoring rubric, were statistically compared to empirical values of writing quality.

\section{Participants}

This study included participants from two 6-month in-service training programs for English teachers in Seoul, South Korea. There were a total of 38 participants, all of whom spoke Korean as their L1. All participants were middle and high school English teachers with extensive language training. They ranged in age from 30 to 56 years old, and had extensive teaching experience that often surpassed 20 years.

\section{Procedure}

To obtain writing samples, the participants were each asked to write an academic paragraph about the cell phone's influence on society. Following the collection of the writings, they were evaluated using a holistic writing rubric designed for the Test of English as a Foreign Language (TOEFL) (Educational Testing Service, 2008). Two native English-speaking EFL instructors independently rated each writing using the holistic rubric. Subsequently, scores were averaged to provide a benchmark for comparison to empirical methods of evaluation. Both instructors had over 10 years of experience as EFL teachers in South Korea, and had received extensive training in EFL assessment at the graduate level.

To empirically determine the quality of each academic paragraph, quantitative strategies for the analysis of cohesion, content, and grammatical accuracy were developed. These strategies are further explained within the following sections.

\section{Empirical Evaluation of Cohesion}

Cohesion, which refers to relationships within a text that make it appear unified, was operationally defined through the work of Halliday and Hasan (1976), which asserts that cohesion is maintained through lexical repetition, reference, conjunctions, ellipsis, and substitution. Lexical repetition was evaluated according to eight categories (Hasan, 1984: p. 202): 

Type
Example
1) Repetition
leave, leaving, left
2) Synonymy
leave, depart
3) Antonymy
leave, arrive
4) Hyponymy
travel, leave/arrive

(leave and arrive are included in the word, travel)

5) Meronymy hand, finger (finger is part of the hand)

6) Equivalence the doctor was their dad

7) Naming the dolphin was named flipper

8) Semblance the girl looked like an angel

According to these categories, examples within texts that used versions of the same word, synonyms, and antonyms; more specific forms of a word, called hyponyms (fork is a hyponym of silverware); constituent parts of a word, called meronyms (finger is a constituent of hand); or seemingly different words to refer to the same object, as in the examples of equivalence, naming, and semblance, were all tallied for each text. The information was then entered into a database for analysis.

In addition to examples of lexical cohesion, conjunctions (e.g., however, and, but, in contrast), which connect different sentences or clauses; references (pronouns and determiners), which denote a semantic relationship to other words within a text; substitution, the insertion of a word or phrase for another; and ellipsis, the omission of a word or phrase, were all tallied for each text (Hoey, 1996).

After empirical values of cohesion were collected for a paragraph, they were divided by the total number of words within the respective paragraph from which they were taken. This ensured that text length did not skew the significance of the empirical values.

\section{Empirical Evaluation of Content, Fluency, and Vocabulary}

Content was empirically evaluated by calculating lexical density, sentence length, the presence of low-frequency vocabulary, and the presence of hard words in each text. First, lexical density, which describes the proportion of content words (nouns, adjectives, verbs, and adverbs) to the total number of words, may reveal how much information is contained within the text (Johansson, 2008). Second, texts containing longer sentences may reveal higher fluency and more sophistication of grammatical features. Third, the frequency of low-frequency vocabulary (vocabulary that appears less often within a corpus) and hard words (words with three or more syllables) may indicate that the student is using more sophisticated vocabulary. While not all long words may be considered difficult (e.g., asparagus), academic texts with longer words may reveal an overall trend toward the use of more sophisticated vocabulary.

Free software programs were used to calculate lexical density, sentence length, vocabulary frequency, and hard words. Sentence length and vocabulary frequency could be determined through using the Lexile Analyzer freely available at lexile.com, while lexical density and hard words were determined by using the free Text Analyser included at usingenglish.com. Since neither of these programs included all of the criteria for evaluating text content, both programs were used.

\section{Empirical Evaluation of Grammar}

Grammar was empirically assessed by tallying each error within a text. Errors were further divided into the following categories for more detailed analysis: prepositions, verb tense/ agreement, count/non-count, plurals/article, run-on sentence, sentence fragment, word form, other.

After empirical values of grammar were collected for a paragraph, they were divided by the total number of words within the respective paragraph from which they were taken. This ensured that text length did not skew the significance of the empirical values.

\section{Data Analysis}

After empirical values for cohesion, content, and grammar were obtained for each academic paragraph, they were used to predict inter-rater writing scores using the multiple regression formula. Issues of multicollinearity were also examined, and texts were qualitatively examined to interpret the significance of the quantitative results.

\section{Results and Discussion}

After rating and averaging writing scores from two raters, scores were compiled into a chart (Appendix A). Although ratings on the TOEFL rubric range from 0 to 5 , inter-rater scores revealed a range from 1.5 to 4.5 . Although writing evaluation by individual raters was generally similar, there were some differences, resulting in a moderate Cronbach's alpha value ( $\alpha$ $=.622$ ). There was a normal distribution of scores along a Gaussian curve, with most of the scores falling between 2.5 to 3.5 .

Comparison of inter-rater scores with empirical values of cohesion, content, and grammar revealed substantially significant results. Using multiple regression, the empirical values of cohesion, content, and grammar correlated highly $(R=.873)$ to inter-rater determinations of writing quality (Table $\mathbf{1}$ ).

ANOVA results further confirm the significance of relationships between variables, yielding an F score of 4.702 which is significant to the .001 probability level. The high correlation between the dependent variable (inter-rater scores) and independent variables (empirical values of cohesion, content, and grammar) suggests that purely quantitative assessments of writing may have a high degree of predictive validity. The $R$-square value further indicates that $76.2 \%$ of the scores assigned through inter-rater evaluation can be explained using the independent variables within this study.

Analysis of individual variables in the multiple regression model yields a more holistic understanding of the results (See Table 2). None of the variables of cohesion (lexical repetition, reference words, or conjunctions) show a significant relationship to inter-rater scores. This may mean that the empirical method for calculating cohesion is problematic. It may also signify that problems with cohesion were not prominent within the texts, which were created by EFL middle and high school English teachers with a great deal of experience. Albeit insignificant, the positive $t$ values for reference words and conjunct-

Table 1.

Multiple regression model summary.

\begin{tabular}{ccccc}
\hline Model & $R$ & $R$ Square & Adjusted $R$ Square & Std. Error of the Estimate \\
\hline 1 & .873 & .762 & .600 & .48086 \\
\hline
\end{tabular}


Table 2.

Independent variables included in the multiple regression analysis.

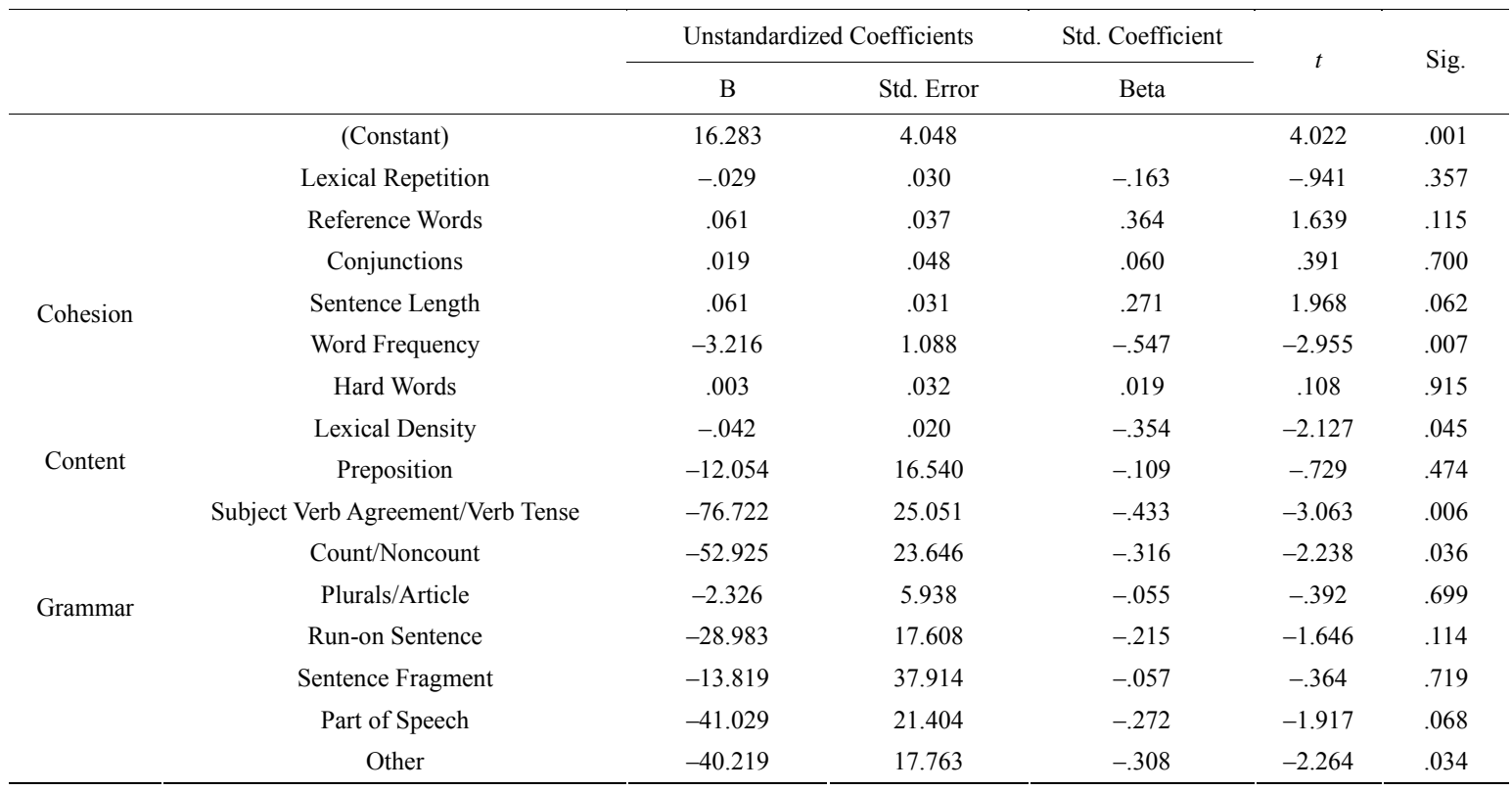

tions $(t=1.639 ; t=.391)$ suggest that these cohesive devices were used more often as writing quality increased; the negative $t$ value for lexical repetition -.941, in contrast, suggests that a larger number of repetition was employed in writings of lower quality. From a qualitative perspective, learners who scored low on the holistic writing rubric appeared to employ simple repetition (e.g., repeating the subject cell phone) much more often than those with a high rubric score. In writings with higher scores, sophisticated references (e.g., this form of technology, a new device) and conjunctions (e.g., Moreover, In contrast, etc.) were used more often than simple repetition. Unlike texts with lower scores, the highest quality texts also included examples of semblance, as in the sentence "A cell phone is a new door to a new era."

In the category of content, both word frequency and lexical density were significantly correlated to inter-rater scores when other independent variables were controlled for, yielding $p$ values of .007 an .045 , respectively. Sentence length, while insignificant at the .05 probability level, was nearly significant $(p=.062)$. In contrast to other variables within the content category, hard words appear to have a miniscule influence on inter-rater scoring, yielding an insignificant score of $p=.915$. Overall, empirical measures of writing seem to accurately predict inter-rater scores. Positive $t$ values of sentence length and hard words $(t=.108 ; t=1.968)$ may suggest that both of these factors increase as writing quality increases, while negative $t$ values of word frequency and lexical density $(t=-2.955 ; t=$ -2.127 ) suggest that both of these factors decrease in intensity as writing quality increases. This supports qualitative observation of texts. Consider the following examples:

1) Participant 6 (Rating 4.5)

Cell phone is a new education tool that leads you to a new educational process. Mobile learning is a topic that people are interested in these days. You can get new information or learn via cell phones from the web as well as from textbooks. Third, it's a new device to get whatever information you need by using wireless connection to the Internet.

\section{2) Participant 12 (Rating 2)}

Cell phone is used for educational purpose for their kids. Cell phone can be books, dictionary, and teachers that give all the information children need all the time. Cell phone can be a toy for their children.

Writings with higher ratings tended to have longer sentences, which were lengthened using sophisticated conjunctions and more difficult vocabulary. In example one, relative clauses, prepositions, and conjunctions are freely employed (e.g., that people, that leads, whatever information, by using, or), thereby lengthening sentences and increasing writing quality. Moreover, this text includes difficult vocabulary, such as device, wireless communication, and mobile, increasing the sophistication of the text. In the second example, conjunctions are hardly used, and sentence length appears to be limited by the proficiency of the learner. The example is more lexically dense because less grammatical features are used to make sophisticated sentences. Simple nouns, such as cell phone, are repeatedly used to convey meaning, without the use of complex grammatical features.

Like content, the grammar category of empirical evaluation has several independent variables that appear to predict interrater scores of participant writings. The subject agreement/tense, countable/uncountable, and miscellaneous "other" categories (this category predominantly contained errors with gerund use), were the most significant predictors of inter-rater writing scores, yielding $p$ values of $.006, .036$, and .034 respectively. Incorrect use of part of speech was nearly significant at $p=.068$. When viewed holistically, trends in grammar use reveal a distinct pattern. Grammatical errors steadily decrease as scores rise from 1.5 to 4.5. Not only do errors within grammatical error categories steadily decrease, but the number of error categories decrease as inter-rater writing scores increase (See Appendix B).

While variables within each category differ in their degree of significance, the overall high correlation of combined variables in the multiple regression model suggests that multiple variables may be involved in the assignment of inter-rater scores. Analysis of multicollinearity further suggests that each factor 
may independently contribute to the assignment of a holistic writing score. All independent variables had tolerance levels above 2 and variance inflation factors (VIF) below 5, suggesting that one factor was not significantly related to another (Appendix C).

Although more study is needed to confirm and increase the predictive validity of variables used within this study, the highly significant results suggest that empirical methods of calculating EFL writing quality may be both a valid and reliable tool for education. The use of empirical methods has several advantages over traditional rubrics. One distinct advantage is that it can reduce subjectivity which is now associated with rubric criteria and rater performance. Empirical methods of writing evaluation, for example, would eliminate the influence of tacit rater biases that linguistically discriminate against cultural or linguistic groups (Johnson \& Van Brackle, 2012).

An additional advantage of discreet empirical criteria for evaluation is the potential for use with automatic grading technology. The use of such technology would greatly increase the potential to provide washback to EFL students anywhere, anytime. Students could use technology to get feedback concerning vocabulary use, grammatical accuracy, or cohesion without the classroom constraints now imposed by instructor-evaluated rubrics.

A final benefit of empirical methods is that they have the potential to provide EFL teacher training. Teachers may obtain valuable feedback concerning their own personal biases employed while assessing writing quality. To facilitate the training process, automatic assessment technology could be used to highlight criteria of evaluation that need to be further emphasized, or deemphasized. Teachers could then learn to provide equal weight to each rubric category being evaluated, regardless of factors such as language, gender, or culture.

\section{Conclusion}

Results of this study reveal that several empirically measurable criteria for writing related to cohesion, content, and grammar can be used to predict overall writing quality of EFL learners. While some of the criteria are more accurate predictors than others, they all appear to synergistically influence the ratings of holistic scores assessed by human raters.

Empirical evaluation of writing has several advantages over traditional methods of evaluation. It allows for the automation of writing assessment, which opens the door to use of the technology as a means of providing both summative and formative writing feedback for students or teachers. Not only can students get more constant and consistent feedback, teachers can receive valuable pre-service or in-service training to sharpen their writing evaluation skills.

Although this study is promising, more study is needed to confirm the validity of empirical measures, as well as to discern additional relevant criteria for empirical writing evaluation of EFL learners. Before such methods of assessment can be used for any summative or formative purpose, they must be thoroughly compared to other forms of writing assessment and examined by a large number of highly trained raters. In addition, empirical methods must be tested with native and non-native English speaking populations to ensure that such techniques are uniformly accurate. Despite the need for further research, the potential to provide automatic EFL writing feedback is clearly evident, and should be further explored.

\section{REFERENCES}

American Council on the Teaching of Foreign Languages (2012). ACTFL proficiency guidelines. URL (last checked 1 October 2012). http://actflproficiencyguidelines2012.org/writing

Beyreli, L., \& Ari, G. (2009). The use of analytic rubric in the assessment of writing performance: Inter-rater concordance study. Educational Sciences: Theory \& Practice, 9, 105-125.

Brown, H. D. (2004). Language assessment principles and classroom practices. White Plains, NY: Pearson Education.

Chodorow, M., \& Burstein, J. (2004). Beyond essay length: Evaluating e-rater's performance on TOEFL essays. Princeton, NJ: ETS.

Cope, B., Kalantzis, M., McCarthey, S., Vojak, C., \& Kline, S. (2011). Technology-mediated writing assessments: Principles and processes. Computers \& Composition, 28, 79-96. doi:10.1016/j.compcom.2011.04.007

Educational Testing Service (2008). TOEFL IBT test: Independent writing rubrics (scoring standards). URL (last checked 1 October 2012). http://www.ets.org/Media/Tests/TOEFL/pdf/Independent_Writing_R ubrics_2008.pdf

Fang, Z., \& Wang, Z. (2011). Beyond rubrics: Using functional language analysis to evaluate student writing. Australian Journal of Language and Literacy, 34, 147-165.

Graham, S., Hebert, M., \& Harris, K. R. (2011). Throw 'em out or make 'em better? State and district high-stakes writing assessments. Focus on Exceptional Children, 44, 1-12.

Halliday, M. A. K., \& Hasan, R. (1976). Cohesion in English. London: Longman.

Hasan, R. (1984). Coherence and cohesive harmony. In J. Flood (Ed.), Understanding reading comprehension (pp. 181-219). Delaware: International Reading Association.

Hoey, M. (1996). Patterns of lexis in text. New York, NY: Oxford University Press.

Hunter, K., \& Docherty, P. (2011). Reducing variation in the assessment of student writing. Assessment \& Evaluation in Higher Education, 36, 109-124. doi:10.1080/02602930903215842

Johansson, V. (2008). Lexical diversity and lexical density in speech and writing: A developmental perspective. Working Papers, 53, 6179.

Johnson, D., \& Van Brackle, L. (2012). Linguistic discrimination in writing assessment: How raters react to African American "errors," ESL errors, and standard English errors on a state-mandated writing exam. Assessing Writing, 17, 35-54. doi:10.1016/j.asw.2011.10.001

Lexile Analyzer [Computer software]. URL (last checked 25 October 2012). http://lexile.com/analyzer

Mansilla, V. B., Duraisingh, E. D., Wolfe, C. R., \& Haynes, C. (2009). Targeted assessment rubric: An empirically grounded rubric for interdisciplinary writing. The Journal of Higher Education, 80, 334353. doi: $10.1353 /$ ihe. 0.0044

Peden, B. F., \& Carroll, D. W. (2008). Ways of writing: Linguistic analysis of self-assessment and traditional assignments. Teaching of Psychology, 35, 313-318. doi:10.1080/00986280802374419

Peregoy, S. F., \& Boyle, O. F. (2005). Reading, writing, and learning in ESL: A resource book for K-12 teachers. New York, NY: Pearson Education.

Schulz, M. M. (2009). Effective writing assessment and instruction for young English language learners. Early Childhood Education Journal, 37, 57-62. doi:10.1007/s10643-009-0317-0

Sévigny, S., Savard, D., \& Beaudoin, I. (2009). Comparability of writing assessment scores across languages: Searching for evidence of valid interpretations. International Journal of Testing, 9, 134-150. doi:10.1080/15305050902880801

Text Analyser [Computer software]. URL (last checked 25 October 2012).

http://www.usingenglish.com/resources/text-statistics.php

Weigle, S. C. (2010). Validation of automated scores of TOEFL iBT tasks against non-test indicators of writing ability. Language Testing, 27, 335-353. doi: $10.1177 / 0265532210364406$ 


\section{Appendix A}

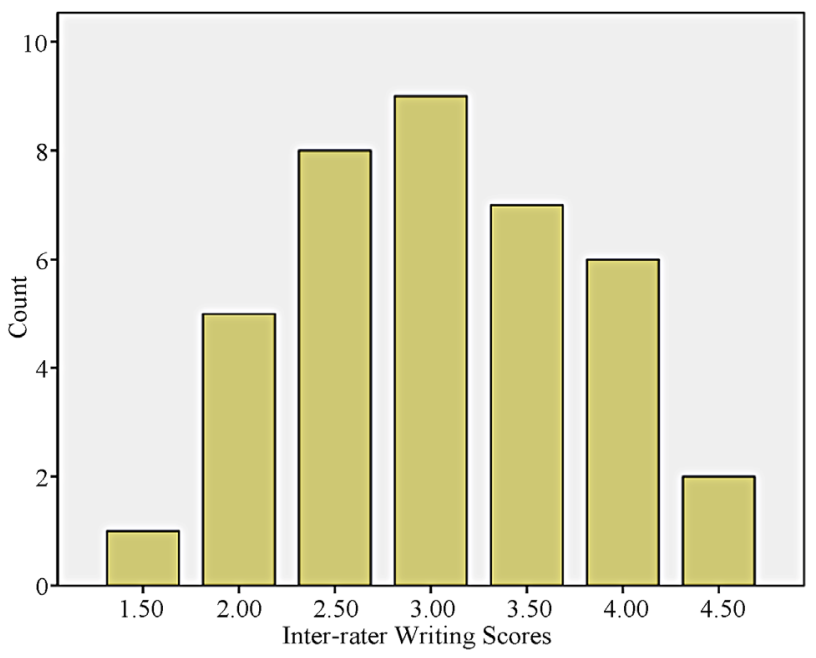

Figure A.

Inter-rater writing score values.

\section{Appendix B}

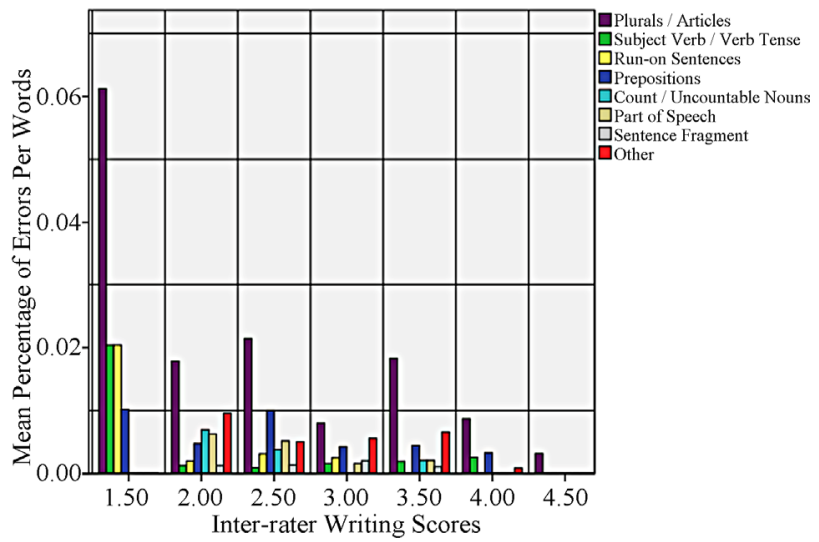

Figure B.

Grammatical errors within writings (separated by score).

\section{Appendix C}

Table C.

Collinearity statistics for independent variables.

\begin{tabular}{cccc}
\hline \multirow{4}{*}{ Cohesion } & & \multicolumn{2}{l}{ Collinearity Statistics } \\
\cline { 3 - 4 } & & Tolerance & VIF \\
& Lexical Repetition & .572 & 1.748 \\
& Reference Words & .315 & 3.170 \\
& Conjunctions & .350 & 2.856 \\
Content & Sentence Length & .391 & 2.559 \\
& Word Frequency & .362 & 2.761 \\
& Hard Words & .219 & 4.564 \\
& Lexical Density & .457 & 2.191 \\
Grammar & Preposition & .486 & 2.057 \\
& Subject Verb Agreement/Verb Tense & .542 & 1.846 \\
& Count/Noncount & .541 & 1.847 \\
& Plurals/Article & .544 & 1.838 \\
& Run-on Sentence & .632 & 1.582 \\
& Sentence Fragment & .438 & 2.281 \\
& Part of Speech & .538 & 1.858 \\
& Other & .582 & 1.718 \\
\hline
\end{tabular}

